\title{
The equivariant integral cohomology ring of the flag manifold of type $C$
}

\author{
Takashi Sato
}

\begin{abstract}
We determine the $T$-equivariant integral cohomology ring of the flag manifold $\operatorname{Sp}(n) / T$ as a quotient ring of a polynomial ring, where $T$ is a maximal torus of $\operatorname{Sp}(n)$ and acts on $\operatorname{Sp}(n) / T$ by left multiplication.
\end{abstract}

\section{Introduction}

Let $G$ be a compact connected Lie group, and let $T$ be a maximal torus of $G$. Goresky, Kottwitz, and MacPherson [GKM] gave a method to determine the $T$ equivariant cohomology ring of the flag manifold $G / T$ combinatorially, where $T$ acts on $G / T$ by left multiplication. They regarded the equivariant cohomology ring $H_{T}^{*}(G / T)=H^{*}\left(E T \times_{T} G / T\right)$ as a subring of the equivariant cohomology ring of the $T$-fixed point set $(G / T)^{T}$ by using the fact that the restriction map $H_{T}^{*}(G / T) \rightarrow H_{T}^{*}\left((G / T)^{T}\right)$ is injective, and they gave a characterization of the elements of the image of the restriction map with complex coefficients. Harada, Holm, and Henriques [HHH] showed that the same characterization holds for the elements of the image with integer coefficients if $G$ is simple and not of type $C$. Guillemin and Zara [GZ] introduced a special graph called a GKM graph which indicates the characterization as a diagram. This method is called the GKM theory. Using the GKM theory, Fukukawa, Ishida, and Masuda [FIM] determined the equivariant integral cohomology rings of flag manifolds of classical type except of type $C$, Fukukawa [F] determined that of the flag manifold of type $G_{2}$, and the author [S1], [S2] determined those of the flag manifolds of type $F_{4}$ and $E_{6}$. In fact, the equivariant cohomology ring of the flag manifold of type $C$ was determined with $\mathbb{Z}\left[\frac{1}{2}\right]$-coefficients in $[\mathrm{FIM}]$. In this article we determine the equivariant integral cohomology ring of the flag manifold of type $C$. Let $T$ be the standard maximal torus of $\operatorname{Sp}(n)$, and let $\left\{t_{i} \mid 1 \leq i \leq n\right\}$ be the standard basis of $H^{2}(B T)$. The main result of this article is the following theorem.

\section{THEOREM 1.1}

The T-equivariant integral cohomology ring of $\mathrm{Sp}(n) / T$ is given as

$$
H_{T}^{*}(\operatorname{Sp}(n) / T) \cong H^{*}(B T)\left[\tau_{i} \mid 1 \leq i \leq n\right] /\left(c_{i}\left(\tau^{2}\right)-c_{i}\left(t^{2}\right) \mid 1 \leq i \leq n\right),
$$

Kyoto Journal of Mathematics, Vol. 56, No. 3 (2016), 681-687

DOI 10.1215/21562261-3600229, (C) 2016 by Kyoto University

Received March 31, 2015. Revised May 21, 2015. Accepted June 1, 2015.

2010 Mathematics Subject Classification: Primary 14M15; Secondary 55N91. 
where $c_{i}\left(x^{2}\right)$ denotes the ith elementary symmetric polynomial in $x_{1}^{2}, \ldots, x_{n}^{2}$ for $x=\tau$ or $t$.

\section{GKM theory}

Let $G$ be a compact connected Lie group, and let $T$ be a maximal torus of $G$. We define the $G K M$ graph of $G / T$ as follows. The GKM graph of $G / T$ is a simple graph whose edges are equipped with elements of $H^{2}(B T)$. The vertex set is the Weyl group $W(G)$ and two vertices $v, v^{\prime} \in W(G)$ are adjacent if and only if there exists a positive root $\alpha$ satisfying $\sigma_{\alpha} v=v^{\prime}$, where $\sigma_{\alpha}$ denotes the reflection associated with $\alpha$. Moreover, for the edge $v v^{\prime}$ satisfying $\sigma_{\alpha} v=v^{\prime}$, we assign the positive root $\alpha \in H^{2}(B T)$, which is called the label of $v v^{\prime}$.

According to [HHH, Theorem 2.3], the restriction

$$
i^{*}: H_{T}^{*}(G / T) \rightarrow H_{T}^{*}\left((G / T)^{T}\right) \cong \prod_{W(G)} H^{*}(B T)
$$

is injective. We often identify $H_{T}^{*}(G / T)$ with the image of $i^{*}$. An element $f$ of $\prod_{W(G)} H^{*}(B T) \cong \operatorname{Map}\left(W(G), H^{*}(B T)\right)$ is called a $G K M$ function if it satisfies the following condition: for any root $\alpha \in \Phi(G)$ and $v \in W(G), f(v)-f\left(\sigma_{\alpha} v\right) \in$ $(\alpha) \subset H^{*}(B T)$, where, for elements $x_{1}, \ldots, x_{n}$ of some ring, $\left(x_{1}, \ldots, x_{n}\right)$ denotes the ideal generated by $x_{1}, \ldots, x_{n}$. When $G$ is simple and not of type $C$, Harada, Henriques, and Holm [HHH, Theorem 3.1 and Lemma 5.2] showed that the subring of $\operatorname{Map}\left(W(G), H^{*}(B T)\right)$ consisting of all GKM functions coincides with the image of $i^{*}: H_{T}^{*}(G / T) \rightarrow \operatorname{Map}\left(W(G), H^{*}(B T)\right)$. Next we define the GKM functions called the equivariant Schubert classes, which give an $H^{*}(B T)$-module basis of $H_{T}^{*}(G / T)$. Recall that for $w \in W(G)$ the length $l(w)$ denotes the number of positive roots which go to negative roots through $w$, and recall that the Bruhat order on $W(G)$ is the reflexive transitive closure of the following relation: for $w \in W(G)$ and a root $\alpha$, if $l\left(\sigma_{\alpha} w\right)=l(w)+1$, then $w \leq \sigma_{\alpha} w$. Let $\Phi^{+}$be the set of all positive roots, and let $\Phi^{-}$be the set of all negative roots. The equivariant Schubert classes $\left\{S_{w}\right\}_{w \in W(G)}$ are defined by the following conditions:

(1) for any $v \in W(G), S_{w}(v)$ is 0 or homogeneous of degree $2 l(w)$,

(2) $S_{w}(w)=\prod_{\alpha \in \Phi^{+} \cap w\left(\Phi^{-}\right)} \alpha$,

(3) if $w \nless v$, then $S_{w}(v)=0$.

The equivariant Schubert classes exist and are unique (cf. [S2, Proposition 3.9 and $3.11]$ ). We obtain the following proposition as an easy consequence of [HHH, Theorem 3.1] and the existence and uniqueness of the equivariant Schubert classes.

\section{PROPOSITION 2.1}

The equivariant Schubert classes $\left\{S_{w}\right\}_{w \in W(G)}$ are contained in the image of $i^{*}: H_{T}^{*}(G / T) \rightarrow \operatorname{Map}\left(W(G), H^{*}(B T)\right)$ and form an $H^{*}(B T)$-basis of $H_{T}^{*}(G / T)$.

Let $\mathcal{G}(G / T)$ be the GKM graph of $G / T$, and let $H^{*}(\mathcal{G}(G / T))$ be the subring of $\operatorname{Map}\left(W(G), H^{*}(B T)\right)$ consisting of all GKM functions. The Weyl group $W(G)$ 
acts on $H^{2}(B T)$ naturally, and we can extend the action onto $H^{*}(B T)$ naturally. Let us introduce the action of the Weyl group $W(G)$ on $H^{*}(\mathcal{G}(G / T))$ : for a GKM function $f$ on $\mathcal{G}(G / T), w \in W(G)$, and a vertex $v$ of $\mathcal{G}(G / T)$, the GKM function $w \cdot f$ is defined by

$$
(w \cdot f)(v)=w\left(f\left(w^{-1} v\right)\right)
$$

It is easily shown that $w \cdot f$ is also a GKM function. For a short while let us assume that $\Phi^{+}$is pairwise relatively prime in $H^{*}(B T)$; that is, for any distinct $\alpha, \beta \in \Phi^{+}, \alpha$ and $\beta$ are relatively prime in $H^{*}(B T)$. For $\alpha \in \Phi(G)$, let us define the divided difference operator $\delta_{\alpha}: H^{*}(\mathcal{G}(G / T)) \rightarrow H^{*}(\mathcal{G}(G / T))$ as follows: for any GKM function $f \in H^{*}(\mathcal{G}(G / T))$,

$$
\delta_{\alpha} f=\frac{1}{\alpha}\left(f-\sigma_{\alpha} \cdot f\right) .
$$

One can easily see that $\delta_{\alpha} f$ is well defined from the formula

$$
f(v)-\left(\sigma_{\alpha} \cdot f\right)(v)=\left(1-\sigma_{\alpha}\right) f(v)+\sigma_{\alpha}\left(f(v)-f\left(\sigma_{\alpha} v\right)\right)
$$

and that it is actually contained in $H^{*}(\mathcal{G}(G / T))$ from the formula

$$
\delta_{\alpha} f(v)-\delta_{\alpha} f\left(\sigma_{\beta} v\right)=\frac{1}{\alpha}\left(f(v)-f\left(\sigma_{\beta} v\right)-\sigma_{\alpha}\left(f\left(\sigma_{\alpha} v\right)-f\left(\sigma_{\sigma_{\alpha} \beta} \sigma_{\alpha} v\right)\right)\right) .
$$

Note that we need the assumption on $\Phi^{+}$for $\delta_{\alpha} f \in H^{*}(\mathcal{G}(G / T))$, and note that $\delta_{\alpha} f$ is contained in $\operatorname{Map}\left(W(G), H^{*}(B T)\right)$ for general $G$. Let $w_{0}$ denote the longest element of $W(G)$. Any element $w \in W(G)$ has the form $w=\sigma_{i_{1}} \cdots \sigma_{i_{k}} w_{0}$, where $\sigma_{i_{j}}$ denotes the reflection associated to the simple root $\alpha_{i_{j}}$ and $l(w)=l\left(w_{0}\right)-k$ (cf. [BGG, Corollary 2.6]). By [S2, Lemma 3.10] we have

$$
\delta_{\alpha} S_{w}= \begin{cases}S_{\sigma_{\alpha} w} & l\left(\sigma_{\alpha} w\right)<l(w) \\ 0 & \text { otherwise. }\end{cases}
$$

This equation holds without the assumption on $\Phi^{+}$. For any $f \in H_{T}^{*}(G / T)$, one can see that $\delta_{\alpha} f$ is also contained in $H_{T}^{*}(G / T)$ since the equivariant Schubert classes form an $H^{*}(B T)$-basis of $H_{T}^{*}(G / T)$. Therefore, we regard $\delta_{\alpha}$ as an operator on $H_{T}^{*}(G / T)$ for general $G$.

\section{PROPOSITION 2.2}

For any root $\alpha$ and $f, g \in H_{T}^{*}(G / T)$, we have $\delta_{\alpha}(f g)=\left(\delta_{\alpha} f\right) g+\left(\sigma_{\alpha} \cdot f\right) \delta_{\alpha} g$.

Proof

By definition, for any vertex $v$, we have

$$
\begin{aligned}
\delta_{\alpha}(f g)(v) & =\frac{1}{\alpha}\left(f g(v)-\sigma_{\alpha}\left(f g\left(\sigma_{\alpha}^{-1} v\right)\right)\right) \\
& =\frac{1}{\alpha}\left(f(v)-\sigma_{\alpha}\left(f\left(\sigma_{\alpha}^{-1} v\right)\right)\right) g(v)+\frac{1}{\alpha}\left(g(v)-\sigma_{\alpha}\left(g\left(\sigma_{\alpha}^{-1} v\right)\right)\right) \sigma_{\alpha}\left(f\left(\sigma_{\alpha}^{-1} v\right)\right) \\
& =\left(\delta_{\alpha} f\right)(v) g(v)+\left(\sigma_{\alpha} \cdot f\right)(v) \delta_{\alpha} g(v) .
\end{aligned}
$$


By Proposition 2.2, one can see that the action of $W(G)$ on $H^{*}(\mathcal{G}(G / T))$ is restricted onto $H_{T}^{*}(G / T)$ from the formula $\sigma_{\alpha} \cdot f=f-\alpha \delta_{\alpha} f$.

\section{The equivariant cohomology ring of $\operatorname{Sp}(n) / T$}

Let $T$ be the standard maximal torus of $\operatorname{Sp}(n)$, and let $\left\{t_{i} \mid 1 \leq i \leq n\right\}$ be the standard basis of the dual of the Lie algebra of $T$. So $\left\{t_{i} \mid 1 \leq i \leq n\right\}$ is a basis of $H^{2}(B T)$. Then the root system $\Phi(\operatorname{Sp}(n))$ is given as

$$
\Phi(\operatorname{Sp}(n))=\left\{ \pm t_{i} \pm t_{j}, \pm 2 t_{k} \mid i \neq j, 1 \leq i, j, k \leq n\right\} .
$$

By identifying $\pm t_{i}$ with $\pm i$, the Weyl group $W(\operatorname{Sp}(n))$ is given as

$$
\begin{aligned}
& W(\operatorname{Sp}(n)) \\
& \quad \cong\{\sigma: \pm[n] \rightarrow \pm[n], \text { bijection } \mid i, j \in \pm[n], \sigma(i)=j \text { implies } \sigma(-i)=-j\},
\end{aligned}
$$

where $\pm[n]=\{ \pm i \mid 1 \leq i \leq n\}$. Note that $W(\operatorname{Sp}(n))$ acts on the Lie algebra of $\operatorname{Sp}(n)$ and that the action is restricted onto $\left\{ \pm t_{i} \mid 1 \leq i \leq n\right\}$. The signed permutation $\sigma$ is uniquely determined by the sequence of its values $\sigma(1), \sigma(2), \ldots, \sigma(n)$. Hence, when we refer to some element $\sigma$ of $W(\operatorname{Sp}(n))$ concretely, we write $\sigma$ as $\sigma(1) \sigma(2) \cdots \sigma(n)$.

For $1 \leq i \leq n$ let us define a GKM function $\tau_{i}$ on the GKM graph of $\operatorname{Sp}(n) / T$ as

$$
\tau_{i}(w)=w\left(t_{i}\right) \quad(w \in W(\operatorname{Sp}(n))) .
$$

For $2 \leq i \leq n$ let $\sigma_{i}$ denote the reflection associated with the simple root $t_{i}-t_{i-1}$, and let $\sigma_{1}$ denote the reflection associated with the simple root $2 t_{1}$. Since

$$
S_{\sigma_{i}}=\sum_{k=i}^{n}\left(t_{k}-\tau_{k}\right)
$$

for any $i$, the $\tau_{i}$ 's are actually contained in $H_{T}^{*}(\operatorname{Sp}(n) / T)$.

The longest element $w_{0}$ of $W(\operatorname{Sp}(n))$ is $-1-2 \cdots-n$, and actually it maps all positive roots to negative roots. By the characterization of the equivariant Schubert classes, the value of $S_{w_{0}}$ is given as

$$
S_{w_{0}}(v)= \begin{cases}\prod_{k=1}^{n}\left(-2 t_{k}\right) \prod_{i<j}\left(-t_{i}-t_{j}\right)\left(-t_{i}+t_{j}\right) & v=w_{0}, \\ 0 & \text { otherwise }\end{cases}
$$

and we can describe $S_{w_{0}}$ concretely as a polynomial in the $\tau_{i}$ 's over $H^{*}(B T)$.

PROPOSITION 3.1

The equivariant Schubert class $S_{w_{0}} \in H_{T}^{*}(\operatorname{Sp}(n) / T)$ is given as

$$
S_{w_{0}}=\prod_{k=1}^{n}\left(\tau_{k}-t_{k}\right) \prod_{i<j}\left(\tau_{i}-t_{j}\right)\left(\tau_{i}+t_{j}\right)
$$

For the proof of Theorem 1.1 we need some algebraic preliminaries. 


\section{DEFINITION 3.2}

A sequence $a_{1}, \ldots, a_{n}$ of elements of a ring $R$ is called regular if, for any $i, a_{i}$ is not a zero divisor in $R /\left(a_{1}, \ldots, a_{i-1}\right)$ and $R /\left(a_{1}, \ldots, a_{n}\right) \neq 0$.

Propositions 3.3 and 3.4 are obvious by definition.

PROPOSITION 3.3

If $a_{1}, \ldots, a_{n}$ is a regular sequence, then so is $a_{1}, \ldots, a_{i-1}, a_{i}+b, a_{i+1}, \ldots, a_{n}$ for $1 \leq i \leq n$ and any $b \in\left(a_{1}, \ldots, a_{i-1}\right)$.

\section{PROPOSITION 3.4}

If $a_{1}, \ldots, a_{n}$ is a regular sequence, then so is $a_{1}, \ldots, a_{i-1}, a_{i+1}, \ldots, a_{n}$ for $1 \leq i \leq n$.

\section{THEOREM 3.5 ([M, THEOREM 17.4(III)])}

Let $(A, \mathfrak{m})$ be a Cohen-Macaulay local ring, and let $a_{1}, \ldots, a_{r}$ be elements of $\mathfrak{m}$. Then the sequence $a_{1}, \ldots, a_{r}$ is regular if and only if the height of the homogeneous ideal $\left(a_{1}, \ldots, a_{r}\right)$ is $r$.

\section{THEOREM 3.6 (CF. [NS, THEOREM 5.5.1])}

Let $F$ be a field, and let $R=F\left[g_{i} \mid 1 \leq i \leq m\right]$ be a nonnegatively graded polynomial ring with degree $\left|g_{i}\right|>0$ for any $1 \leq i \leq m$. Assume that $a_{1}, \ldots, a_{n}$ is a regular sequence in $R$, which consists of homogeneous elements of positive degree. Then the Poincaré series of $R /\left(a_{i} \mid 1 \leq i \leq n\right)$ is given as

$$
\frac{\prod_{i=1}^{n}\left(1-x^{\left|a_{i}\right|}\right)}{\prod_{i=1}^{m}\left(1-x^{\left|g_{i}\right|}\right)} .
$$

Proof

For a nonnegatively graded $F$-module $M$ of finite type, let $P(M, x)$ denote the Poincaré series of $M$, namely,

$$
P(M, x)=\sum_{n=0}^{\infty}\left(\operatorname{dim}_{F} M_{n}\right) x^{n},
$$

where $M_{n}$ denotes the degree $n$ part of $M$. Then obviously we have

$$
P(R, x)=\frac{1}{\prod_{i=1}^{m}\left(1-x^{\left|g_{i}\right|}\right)} .
$$

Since $a_{1}, \ldots, a_{n}$ is a regular sequence, the multiplication by $a_{i}$ induces an injection on a graded $F$-module $R /\left(a_{1}, \ldots, a_{i-1}\right)$. Therefore,

$$
P\left(R /\left(a_{1}, \ldots, a_{i}\right), x\right)=\left(1-x^{\left|a_{i}\right|}\right) P\left(R /\left(a_{1}, \ldots, a_{i-1}\right), x\right) .
$$

The induction on $i$ completes the proof. 
Proof of Theorem 1.1

Since divided difference operators decrease the degree by two, any divided difference operator maps $\tau_{i}$ 's and $t_{i}$ 's to constant functions. Hence, by Proposition 2.2, Proposition 3.1, and (2.1), all equivariant Schubert classes are written as a polynomial in the $\tau_{i}$ 's over $H^{*}(B T)$. Therefore, by Proposition 2.1, $H_{T}^{*}(\operatorname{Sp}(n) / T)$ is generated by the $\tau_{i}$ 's as an $H^{*}(B T)$-algebra.

Since $W(\operatorname{Sp}(n))$ is the signed permutation group on $n$ letters, for $1 \leq i \leq n$ we have $c_{i}\left(\tau^{2}\right)-c_{i}\left(t^{2}\right)=0$ as a GKM function. The natural surjection $H^{*}(B T)\left[\tau_{i} \mid\right.$ $1 \leq i \leq n] \rightarrow H_{T}^{*}(\operatorname{Sp}(n) / T)$ factors through $H^{*}(B T)\left[\tau_{i} \mid 1 \leq i \leq n\right] /\left(c_{i}\left(\tau^{2}\right)-\right.$ $\left.c_{i}\left(t^{2}\right) \mid 1 \leq i \leq n\right)$. We will show that $H^{*}(B T)\left[\tau_{i} \mid 1 \leq i \leq n\right] /\left(c_{i}\left(\tau^{2}\right)-c_{i}\left(t^{2}\right) \mid\right.$ $1 \leq i \leq n)$ is a free $\mathbb{Z}$-module and has the same rank with $H_{T}^{*}(\operatorname{Sp}(n) / T)$ at each degree by an argument of regular sequences. Then we will see that the surjective homomorphism

$$
H^{*}(B T)\left[\tau_{i} \mid 1 \leq i \leq n\right] /\left(c_{i}\left(\tau^{2}\right)-c_{i}\left(t^{2}\right) \mid 1 \leq i \leq n\right) \rightarrow H_{T}^{*}(\operatorname{Sp}(n) / T)
$$

is an isomorphism.

Let us show that the sequence

$$
c_{1}\left(\tau^{2}\right)-c_{1}\left(t^{2}\right), c_{2}\left(\tau^{2}\right)-c_{2}\left(t^{2}\right), \ldots, c_{n}\left(\tau^{2}\right)-c_{n}\left(t^{2}\right)
$$

is a regular sequence in $H^{*}(B T)\left[\tau_{i} \mid 1 \leq i \leq n\right] \otimes(\mathbb{Z} / p \mathbb{Z})$ for any prime number $p$. Then, by Theorem 3.6, we will see that the Poincaré series of $H^{*}(B T)\left[\tau_{i} \mid 1 \leq i \leq\right.$ $n] /\left(c_{i}\left(\tau^{2}\right)-c_{i}\left(t^{2}\right) \mid 1 \leq i \leq n\right) \otimes(\mathbb{Z} / p \mathbb{Z})$ does not depend on $p$. Hence, $H^{*}(B T)\left[\tau_{i} \mid\right.$ $1 \leq i \leq n] /\left(c_{i}\left(\tau^{2}\right)-c_{i}\left(t^{2}\right) \mid 1 \leq i \leq n\right)$ must be free because it is of finite type.

By Propositions 3.3 and 3.4, it is sufficient to show that the sequence

$$
t_{1}, t_{2}, \ldots, t_{n}, c_{1}\left(\tau^{2}\right), c_{2}\left(\tau^{2}\right), \ldots, c_{n}\left(\tau^{2}\right)
$$

is regular in $H^{*}(B T)\left[\tau_{i} \mid 1 \leq i \leq n\right] \otimes(\mathbb{Z} / p \mathbb{Z})$. Since the ordinary cohomology $H^{*}(\operatorname{Sp}(n) / T) \cong \mathbb{Z}\left[\tau_{i} \mid 1 \leq i \leq n\right] /\left(c_{i}\left(\tau^{2}\right) \mid 1 \leq i \leq n\right)$ (cf. [B, Proposition 30.2]) is finite, one can see that the height of $\left(c_{i}\left(\tau^{2}\right) \mid 1 \leq i \leq n\right)$ is $n$ by dimensional reasoning. Since a polynomial ring over a field is Cohen-Macaulay, by Theorem 3.5, the sequence $c_{1}\left(\tau^{2}\right), \ldots, c_{n}\left(\tau^{2}\right)$ is regular in $\mathbb{Z} / p \mathbb{Z}\left[\tau_{i} \mid 1 \leq i \leq n\right]$ for any $p$. Hence, (3.2) is a regular sequence.

By Theorem 3.6, the Poincaré series of the graded $\mathbb{Z} / p \mathbb{Z}$-module $H^{*}(B T)\left[\tau_{i} \mid\right.$ $1 \leq i \leq n] /\left(c_{i}\left(\tau^{2}\right)-c_{i}\left(t^{2}\right) \mid 1 \leq i \leq n\right) \otimes(\mathbb{Z} / p \mathbb{Z})$ is given as

$$
\frac{\prod_{i=1}^{n}\left(1-x^{2 i}\right)}{\left(1-x^{2}\right)^{2 n}},
$$

and it does not depend on $p$ and coincides with the Poincaré series of the graded $\mathbb{Z}$-module $H^{*}(B T)\left[\tau_{i} \mid 1 \leq i \leq n\right] /\left(c_{i}\left(\tau^{2}\right)-c_{i}\left(t^{2}\right) \mid 1 \leq i \leq n\right)$. By the Serre spectral sequence of the fibration $\operatorname{Sp}(n) / T \rightarrow E T \times_{T} \mathrm{Sp}(n) / T \rightarrow B T$, one can see that the Poincaré series of $H_{T}^{*}(\operatorname{Sp}(n) / T)$ is the product of those of the graded $\mathbb{Z}$-modules $H^{*}(B T)$ and $H^{*}(\operatorname{Sp}(n) / T) \cong \mathbb{Z}\left[\tau_{i} \mid 1 \leq i \leq n\right] /\left(c_{i}\left(\tau^{2}\right) \mid 1 \leq i \leq n\right)$, and it coincides with (3.3). 


\section{References}

[BGG] I. N. Bernšteĭn, I. M. Gel'fand, and S. I. Gel'fand, Schubert cells, and the cohomology of the spaces G/P (in Russian), Uspekhi Mat. Nauk 28 (1973), no. 3, 3-26. MR 0429933.

[B] A. Borel, Sur la cohomologie des espaces fibrés principaux et des espaces homogènes de groupes de Lie compacts, Ann. of Math. (2) 57 (1953), 115-207. MR 0051508.

[F] Y. Fukukawa, The cohomology ring of the GKM graph of a flag manifold of type $G_{2}$, preprint, arXiv:1207.5229v1 [math.AT].

[FIM] Y. Fukukawa, H. Ishida, and M. Masuda, The cohomology ring of the GKM graph of a flag manifold of classical type, Kyoto J. Math. 54 (2014), 653-677. MR 3263556. DOI 10.1215/21562261-2693478.

[GKM] M. Goresky, R. Kottwitz, and R. MacPherson, Equivariant cohomology, Koszul duality, and the localization theorem, Invent. Math. 131 (1998), 25-83. MR 1489894. DOI 10.1007/s002220050197.

[GZ] V. Guillemin and C. Zara, 1-skeleta, Betti numbers, and equivariant cohomology, Duke Math. J. 107 (2001), 283-349. MR 1823050.

DOI 10.1215/S0012-7094-01-10724-2.

[HHH] M. Harada, A. Henriques, and T. S. Holm, Computation of generalized equivariant cohomologies of Kac-Moody flag varieties, Adv. Math. 197 (2005), 198-221. MR 2166181. DOI 10.1016/j.aim.2004.10.003.

[M] H. Matsumura, Commutative Ring Theory, Cambridge Stud. Adv. Math. 8, Cambridge Univ. Press, Cambridge, 1986. MR 0879273.

[NS] M. D. Neusel and L. Smith, Invariant Theory of Finite Groups, Math. Surveys Monogr. 94, Amer. Math. Soc., Providence, 2002. MR 1869812.

[S1] T. Sato, The T-equivariant integral cohomology ring of $F_{4} / T$, Kyoto J. Math. 54 (2014), 703-726. MR 3276414. DOI 10.1215/21562261-2801786.

[S2] The T-equivariant integral cohomology ring of $E_{6} / T$, preprint, arXiv:1406.3893v1 [math.AT].

Department of Mathematics, Kyoto University, Kyoto, Japan; 